\title{
Design, simulation and manufacturing a CFRP prototype mirror for active/adaptive optics
}

Baghsiahi, Hadi, Jones, Martyn, Brooks, David, Doel, Peter

Hadi Baghsiahi, Martyn Jones, David Brooks, Peter Doel, "Design, simulation and manufacturing a CFRP prototype mirror for active/adaptive optics," Proc. SPIE 11175, Optifab 2019, 111750W (15 November 2019); doi: 10.1117/12.2537775

SPIE. Event: SPIE Optifab, 2019, Rochester, New York, United States 


\title{
Design, Simulation and Manufacturing a CFRP Prototype Mirror for Active/Adaptive optics
}

\author{
Hadi Baghsiahi*a, Martyn Jones ${ }^{\mathrm{b}}$, David Brooks ${ }^{\mathrm{a}}$, Peter Doel ${ }^{\mathrm{a}}$ \\ ${ }^{\mathrm{a}}$ Dept of Physics and Astronomy, University College London, London, UK; ${ }^{\mathrm{b}}$ Composite Centre, \\ Glyndwr University, UK.
}

\begin{abstract}
In this work, we are aiming to reduce the mass of large precision mirrors for space missions by using space approved composite materials. We report the development of an active/adaptive optics prototype of carbon fiber reinforced polymer mirrors using a pre-impregnated (pre-preg) composite MTM44-1/IMS65. The carbon fiber mirror has 16 layers of carbon fiber and one layer of polishable resin which compensate the well-known problem of "fiber print through". The development of a fabrication method, suited for creating a CFRP mirror is outlined. As all materials will change properties to some degree, during cool down to cryogenic temperatures, FEA model is employed to investigate the change on overall form and figure of the mirror. Characterizing this dimensional change is critical in insuring that any mirror material can be used in this environment and, if required, corrected either by utilizing a deformable mirror control systems or by correction in the optical system. Two different piezoelectric actuators are modelled and used to create an active composite reflector. Push actuators and micro fiber composite (MFC) actuators are simulated and the performance of them are compared by surface deformation and dynamic response.
\end{abstract}

Keywords: CFRP, Carbon fibre, large mirror, space telescopes, composite mirrors, CTE, active optics, adaptive optics, aberration corrections.

\section{INTRODUCTION}

The differential expansion and contraction of the matrix and fibres in a carbon fibre layer, during cure and operation, causes internal strain resulting in distortion. The opportunity to create a mirror substrate with a high specific stiffness is attractive to many industries and research sectors. Composite materials offer low mass and tailorable properties and are perfectly suited to a wide variety of applications. In studies by Doel et al at UCL, composite mirrors have been created using a CFRP substrate that is fully coated in Nickel at a thickness of $50 \mu \mathrm{m}$ [1]. The purpose of the mirror demonstrator was to create an active deformable mirror to be used in a large ground-based telescope. The Planck telescope, launched alongside the Herschel Space Observatory, used a CFRP sandwich structure to observe $0.3-10 \mathrm{~mm}$ range at 40K [2]. Due to the wavelength observed, the surface figure and form accuracy are factors less than required for the far infrared. However, the technologies used in Planck should be considered in future missions. The CFRP face plates and the core were made from the same material, eliminating differential CTE effects in the sandwich [3]. Additionally, the CFRP face plates were fabricated using an automated tape layup procedure, improving the ply orientation accuracy and removing the variability associated with human error [4]. In a high proportion of literature for space structures and mirror substrates using PMCs, cyanate ester resin seemed to be used extensively [5]. The James Webb Space Telescope uses a composite of cyanate ester resin as its matrix with carbon fibre reinforcements for its structural components [6].

The current state of the art method for manufacturing method uses a polished mandrel to replicate the mirror, this is the method used for this project. However, some polishing needs to be performed in an attempt to further improve the surface roughness for a sample created. Another problem and drawback in using CFRP in large telescope structure is the fibre print through which makes the surface rough and also creates periodic structure which can cause diffraction. The most common solution to eliminate fibre print through is to include a resin rich layer on the reflective surface of the mirror. This resin rich layer can be conventionally polished, diamond turned, or if a replication method is used, will already have surface that satisfies the figure error limit. A resin layer of approximately $0.25 \mathrm{~mm}$ is required to eliminate the effect of fibre print through [7]. Consideration must be taken as to the operating conditions of the mirror. In a paper by Kim, fibre print through mitigation using a resin rich layer can cause high special frequency errors due to differential thermal expansion of the substrate and the resin layer [8]. This means that the radial stiffness of the mirror needs to be matched in order to alleviate this error.

Optifab 2019, edited by Blair L. Unger, Jessica DeGroote Nelson, Proc. of SPIE Vol. 11175,

$111750 \mathrm{~W} \cdot$ · 2019 SPIE · CCC code: 0277-786X/19/\$21 · doi: 10.1117/12.2537775 


\section{MIRROR FABRICATION}

For the fabrication of the mirror a replication process is used, where the unidirectional pre-preg carbon fiber layers are stacked at a designed angle on the top of a mandrel. The mandrel had been previously polished to the required surface quality. Several different designs were used but the two main designs had 16 and 32 layers. Figure 1 and Figure 2 shows the process of replication process and the final product after the mirror has been cut and trimmed using water jet cutting.
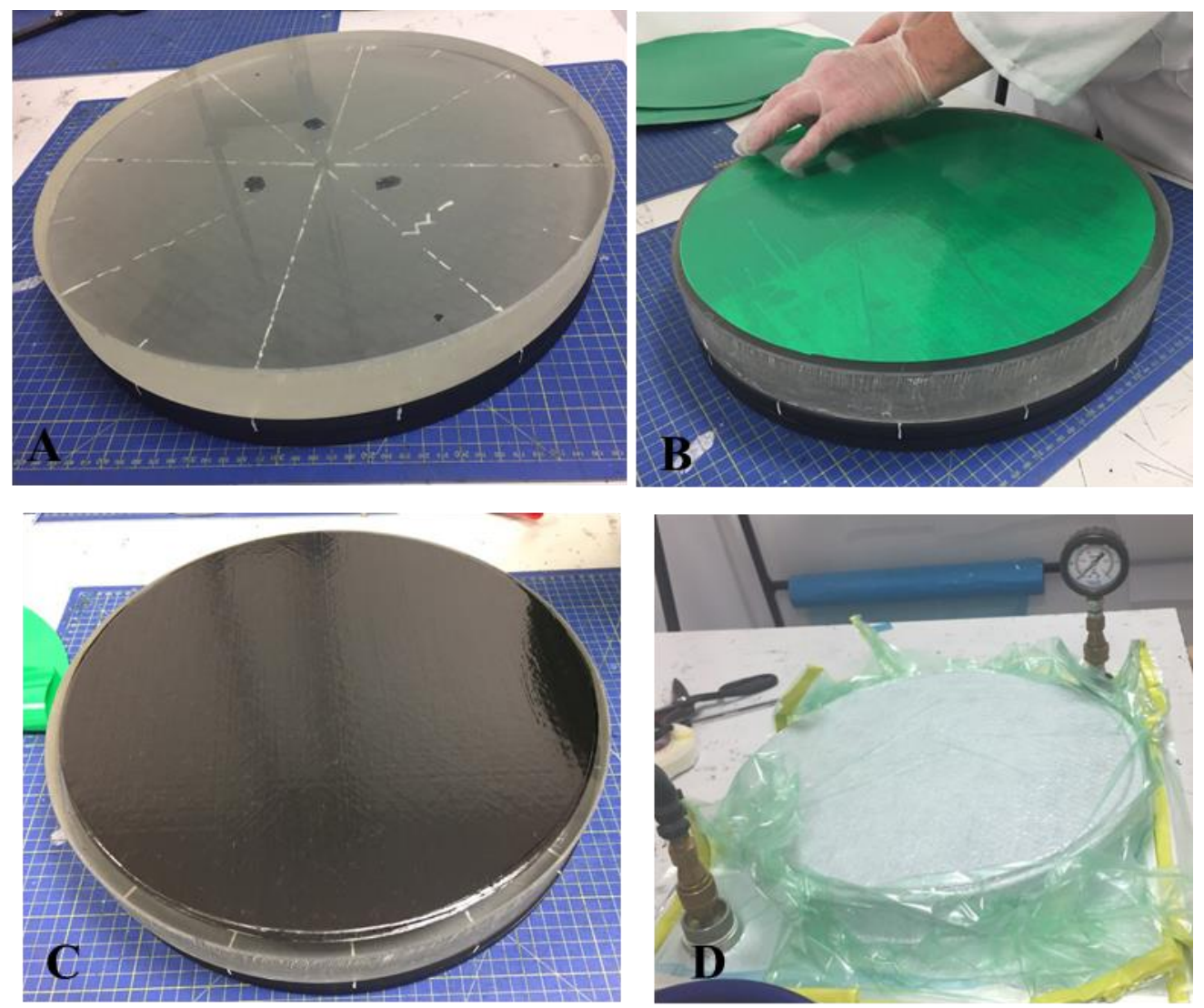

Figure 1: Fabrication process. A: Mandrel and the measuring table, B: A raw layer of carbon fibre. C: 16 layers of carbon fibre stacked. D: Vacuumed and ready to go to Autoclave

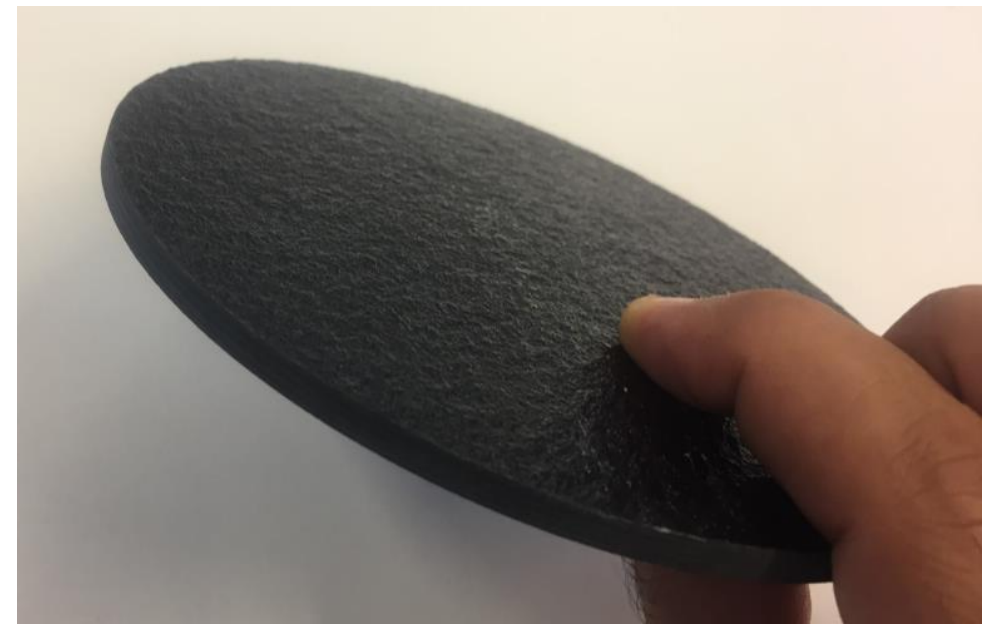

Figure 2:100 mm dimeter sample after autoclave and cut by water jet cutting. 


\subsection{Material selection}

After extensive research, MTM44-1 was chosen for the mirror substrate material. MTM44-1 is an epoxy resin matrix prepreg that is widely used in space structures. It has low thermal shrinkage during cure and is NASA space qualified to eliminate outgassing. MTM44-1 comes in an array of pre-impregnated sheets with unidirectional or bidirectional fibre architecture. Different carbon fibre types, strengths and tow thickness are also available.

Ideally a carbon fibre reinforced cyanate ester would have been used. Cyanate esters tend to have a much lower CTE and cure shrinkage with the only real difficulty occurring due to moisture uptake, which can be controlled. However these materials are very difficult to source. The manufacturing process for producing CFRP materials is most efficient when producing items in bulk. As the dominant market for composites is the aerospace sector, who mainly use epoxy based matrix materials, cyanate ester matrix composites are rarely run.

Table 1: Comparison of properties of different materials used for large telescope mirrors.

\begin{tabular}{|l|l|l|l|}
\hline Material & Density & Young's modulus & CTE \\
\hline $\mathrm{Be}$ & 1.85 & 303 & 11.4 \\
\hline $\mathrm{Al}$ & 2.73 & 71 & 24 \\
\hline Zerodur & 2.57 & 89 & 0.02 \\
\hline $\mathrm{C} /$ Sic composite & 2.8 & 320 & 2.3 \\
\hline $\mathrm{CFRP}$ & 1.5 & 120 & 0.03 \\
\hline
\end{tabular}

Table 1 shows the mechanical and thermal properties of materials unitised in space optics. Low CTE and high stiffness is always desirable. The stiffness of the material is paramount to ensure that the mirror substrate can withstand polishing loads, launch loads and thermal gradients during cool down and operation. A parametric study was undertaken to ascertain the optimum number of plies (and similarly the mirror thickness) for the mirror substrate. For quasi-isotropic, symmetric layups it is important that the number of plies is devisable by 4 and is symmetric about the mid plane.

\subsection{Mandrel selection}

The replication requires the composite to be cured on a mandrel and upon release will replicate the form of the mandrel. With this in mind a mandrel was purchased, from which the CFRP mirror was to be replicated. There were two options when selecting a mandrel material, glass, aluminium. Key to the material selection is the stability of the mandrel over the cure temperature range. Materials with high CTE will expand and change their form during curing. Aside from changing the shape of the final mirror, it will also cause residual stress to build in the mirror, further increasing the change in mirror form. Based on the availability in the project we have chosen glass material over aluminium due to CTE match with the carbon fibre material to avoid distortion on the mirror surface during the curing process.

\subsection{Release agent}

A release agent, in this case a polymer based liquid Frekote 44-NC, was used to create a barrier between the mandrel and the composite whilst it cured. This barrier prevents the epoxy resin bonding to the glass and is a necessity when fabricating composites.

\section{RESULTS AND DISCUSSION}

Fibre print through is a well known issue in the fabrication of carbon fibre mirrors and can make the surface uneven and create a periodic structure, this could cause diffraction. Figure 3 shows the fibre print through on of our CFRP sample under atomic force microscope (AFM). To eliminate fibre print through we include a resin rich layer on the reflective surface of the mirror. This resin rich layer should be conventionally polished, diamond turned, or if a replication method is used, will already have surface that satisfies the figure error limit. A resin layer of approximately $0.25 \mathrm{~mm}$ is required to eliminate the effect of fibre print through. Consideration must be taken as to the operating conditions of the mirror. We used a resin layer as shown in Figure 5 (left) to cover the fibres and achieve a surface with better optical quality. This work is still under investigation and we will use polishing to enhance the resin surface before metal coating. 


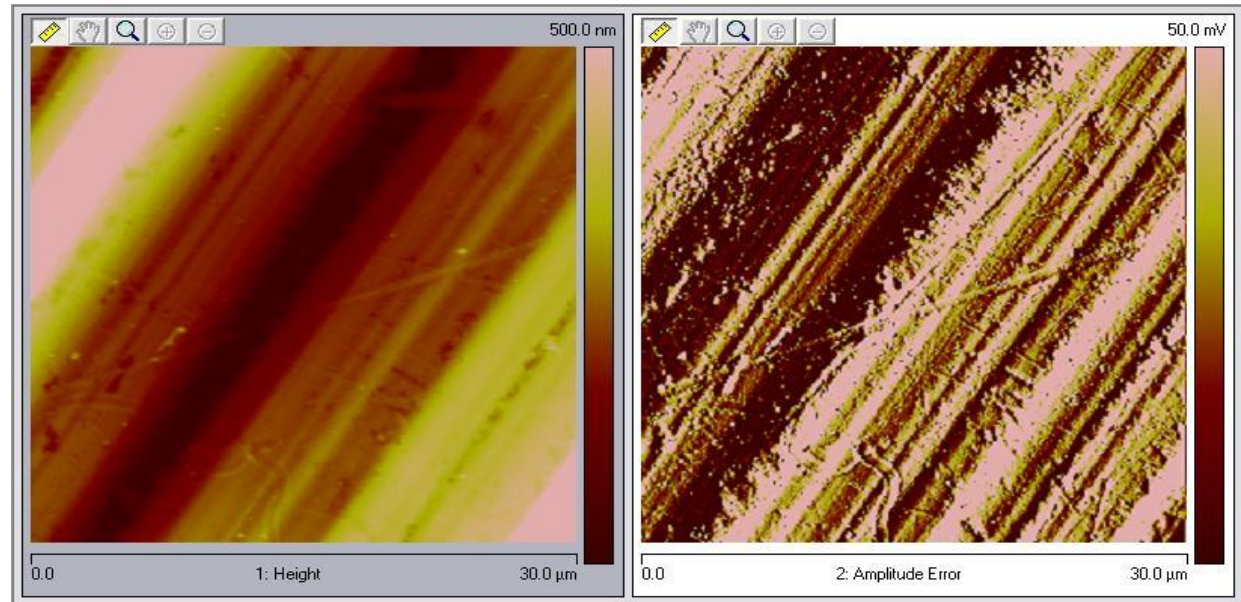

Figure 3: Pictured from the CFRP surface measured using AFM. The results shows the fibre print through and a surface roughness of $200 \mathrm{~nm}$.

In order to ascertain how the thermal gradient of the operating temperature effect and the form of the mirror a finite element model in COMSOL was developed. This model uses the geometry in the replication process and the coefficient of expansion and other material properties for MTM44-1. The initial baseline models used gravitational loads to define the number of plies the substrate should have to provide the minimal deflection during fabrication and polishing.

Although larger than the final required surface error it is worth noting that this surface error could be easily rectified by corrective polishing of the resin rich face. Below are the results of the simulation for using different numbers of layers in the mirror layup and the effects of the temperature on the substrate. Low temperatures were used in the simulation to find the form error and distortion of the material and mirror surface.

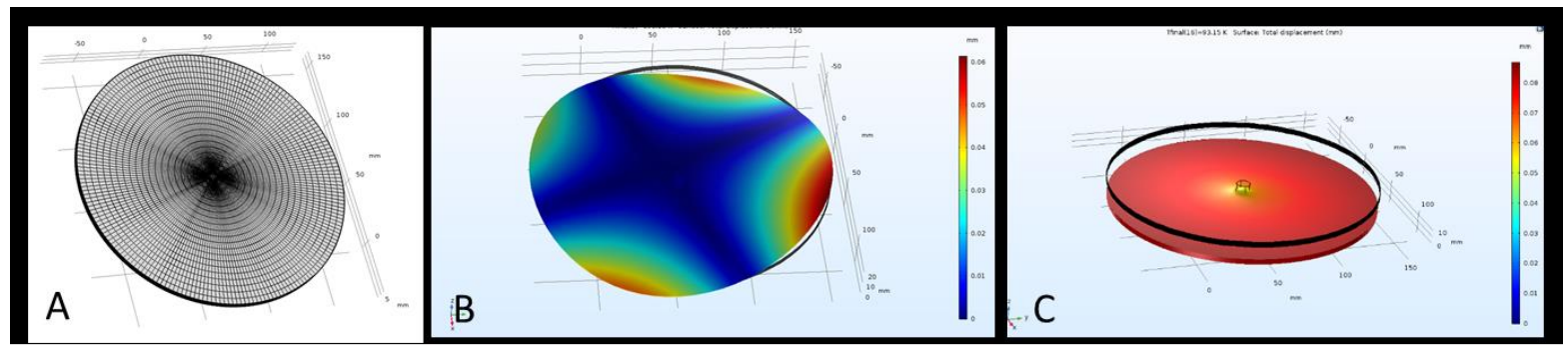

Figure 4: A model of CFRP distortion with different layers. A: meshing figure. B: 4 layers. C: 16 layers

All materials will change properties to some degree during the cool down to cryogenic temperatures. This deformation needs to be compensated by utilizing deformable mirror control systems or by correction in the optical system. However, for secondary correction to be utilized the deformation must not only be predictable, it must also be repeatable. We modelled and experimentally checked the surface of the mirror for active and adaptive optics. In this method, push/pull actuators and macro fiber composite (MFC) actuators are used and compared to change the surface of the mirror to correct for atmospheric aberrations. Figures 5, 6 and 7 show some results from the experiment and simulation in COMSOL. 

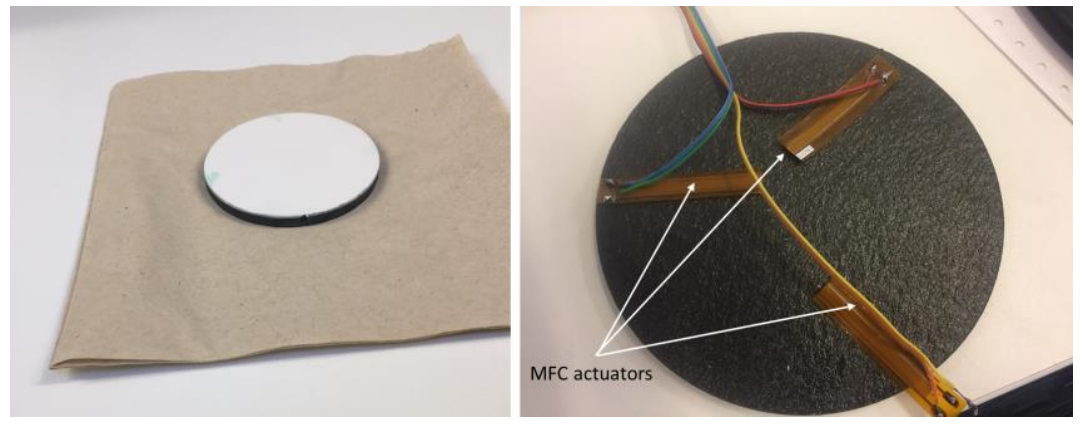

Figure 5: Left: prepared sample of CFRP with a resin layer on the top. Right: preparing the back structure for testing MFC actuators.
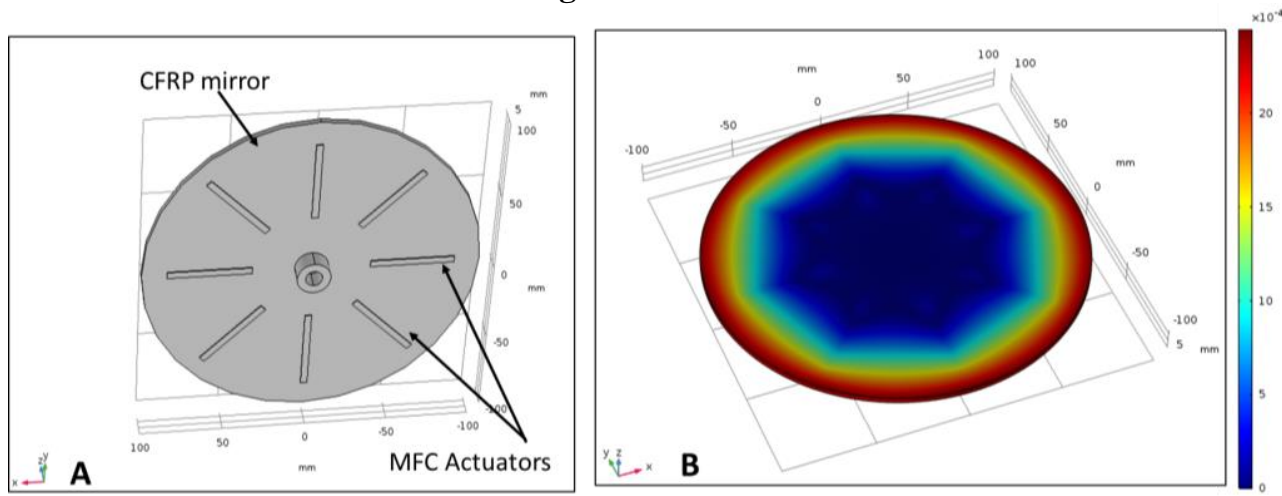

Figure 6: MFC actuators on CFRP design and the FEA results after applying voltage.
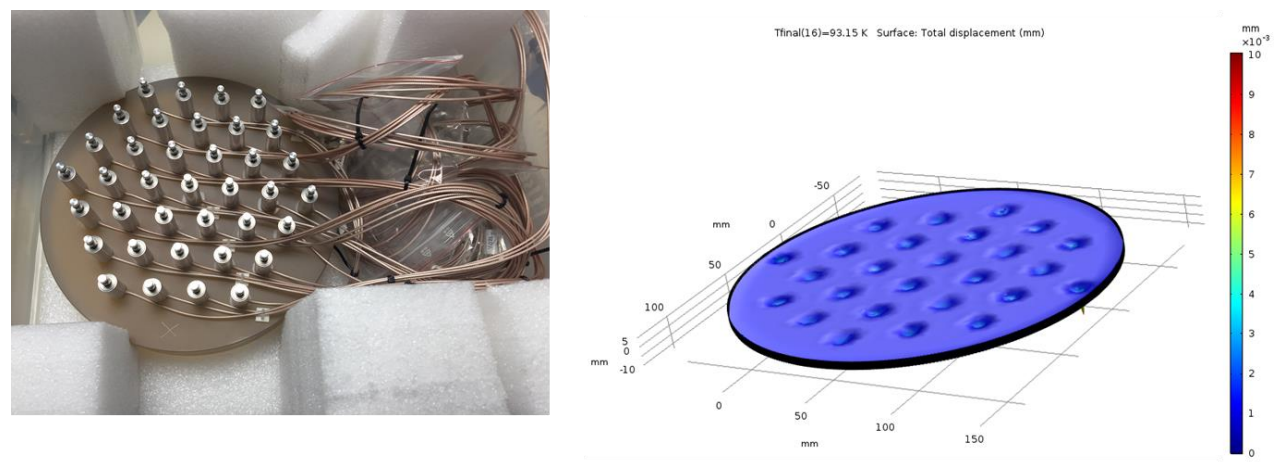

Figure 7: Push actuator structure and the FEA results.

Figure 6 and Figure 7 compare the different types of backing structure design required and highlight the size of the MFC and push/pull actuators. MFC's, which are glued to the back of the mirror are very thin and light, whilst push/pull actuators need a bulky and rather heavy backing structure to work. However, the MFC's require a much larger voltage compared to push/pull actuators to be effective. Push/pull actuators create a point force that will create a Gaussian shaped deformation on the surface. This force and deformation can be controlled and applied to a particular area of the mirror. In contrast, the MFC shows a wider change on the surface as they act on an area rather a point. Therefore, careful design is needed to achieve the required shape on the entire surface due to the overlap of different surface deformations. 


\section{CONCLUSION}

To conclude, design and manufacturing of a carbon fiber composite mirror prototype was investigated and we found that a minimum of 16 layers of carbon fiber layers are needed to achieve a rigid and stable structure after autoclave curing. 32 layers are recommended for the structures without active and adaptive optics but in case of using actuators the rigidity of the mirror might be more than what can be deformed by the actuators. Push actuators and MFC actuators have their advantages and disadvantages. Push actuators apply point forces and are easier to control but they are bulky, heavy and more expensive. In addition, they apply the force in one point, which can create damage to surface such as micro cracking, and delamination, which will damage the mirrors and cause aging. MFC actuators are light and easy to use but they need a high voltage to operate and the strength of the adhesive material between the mirror and MFC might become poor over time.

\section{REFERENCES}

[1] Thompson, S, Brooks, D, Doel, P. "A nickel-carbon-fibre composite for large adaptive mirrors: Fabrication methods and properties", Opt. Express 16, 1321-1330 (2008).

[2] Catanzaro, B. Thomas, J. Backovsky. S, Barber, D. Small, D. Johnston, R. Cohen, E. "Effects of aberrations (low order and quilting) on the performance of the all-composite design for the Herschel Space Observatory". Proc. SPIE 4444, Optomechanical Design and Engineering (2001).

[3] Delouard, P. Krahenbuhl, U. Peikert, G. "Materials characterisation at cryogenic temperatures for the Planck telescope", Proceedings of the European Conference on Spacecraft Structures, Materials and Mechanical Testing 2005.

[4] Arao, Y. Koyanagi, J. Utsunomiya, S. and Kawada, H. "Effect of ply angle misalignment on out-of-plane deformation of symmetrical cross-ply CFRP laminates: Accuracy of the ply angle alignment," Compos. Struct. 93(4), 1225-1230 (2011).

[5] Polis, D. Sovinski, M. Harris, B. Puckett, D. "Cryogenic Durability of a carbon fibre reinforced cyanate ester resin composite: Degree of cure" NASA Goddard Space Flight Centre (2006).

[6] Gardner, JP. "The Scientific Capabilities of the James Webb Space Telescope" Proc. of SPIE, 7010-19, (2008).

[7] Massarello, J. Welsh, J. Hochhalter, J. Maji, A. Fuierer, P. "Fibre print-through mitigation technique for composite mirror replication", Optical Engineering, v 45, n 12, December, 2006, p 123401

[8] Kim, K. Hale, R. "Analysis of Surface Deformation Due to Thermal Load on Circular Quasi-Isotropic Laminate Mirrors", Structural Dynamics, and Materials Conf. Schaumburg, Illinois, AIAA Paper 2008-2185, 7-10 Apr. 2008. 